\title{
Wieder zurück? Die SPD als Volkspartei
}

\author{
von Franz Walter und Matthias Micus
}

Die SPD befindet sich seit vier Jahrzehnten in einer zunächst unbemerkten Krise, die ihren Status als Volkspartei zunehmend unterminiert. Seit 1973 gelingt es den Sozialdemokraten nur noch selten, den Integrationsbogen zwischen ihren auseinanderdriftenden Anhängergruppen zu spannen. Dabei verliert die SPD Unterstützung in alle Richtungen, zuletzt nicht mehr nur an den Rändern, sondern ganz massiv auch bei ihrer Kernklientel. In der Folgezeit hat sich die Partei strukturell stark verengt, doch zeichneten sich zuletzt Möglichkeiten zur Umkehr des Niederganges ab - sowohl auf der organisatorischen Ebene als auch im inhaltlich-programmatischen Bereich.

For almost four decades, the German Social Democratic Party (SPD) has been in an initially unnoticed state of crisis, gradually undermining its catch-all status. Ever since 1973, the Social Democrats have rarely succeeded in balancing their diverging constituencies. As a matter of fact, the SPD is losing out on all fronts, not only on the fringes of its electoral spectrum but also regarding its core followers. The Party has structurally constricted itself to a large extent. Nonetheless, the SPD is not left without opportunities to reverse the process of its decline - both in organisational and programmatic terms.

\section{Einleitung}

Die Zahl der Nekrologe auf die Sozialdemokratie ist nicht gering. Bekanntlich verabschiedete bereits 1983 Ralf Dahrendorf - mit beträchtlicher Resonanz und Wirksamkeit im politischen Kommentar - das Zeitalter der Sozialdemokratie. ${ }^{1}$ Sie habe erreicht, schrieb der Lord und Soziologe seinerzeit, was sie ursprünglich als Sozialbewegung angetrieben hatte - und sei infolgedessen aufgrund der höchst erfolgreichen Resultate ihres Tuns überflüssig geworden. Dahrendorf gab den Ton vor; unzählige Interpreten sind ihm gefolgt.

\section{Das Jahr 1973 als Wendepunkt}

Nun war Dahrendorf ein unzweifelhaft kluger Kopf, dessen Argumentation sich schwerlich als abstruse Fabuliererei abtun ließ. ${ }^{2}$ Doch spricht viel dafür, die

1 Vgl. Dahrendorf, R.: Die Chancen der Krise. Über die Zukunft des Liberalismus, Stuttgart, 1983, $16 \mathrm{ff}$.

2 Zu Dahrendorf vgl. die Studie von Micus, M.: Ralf Dahrendorf. Scheitern eines Experiments, in: Lorenz, R./Micus, M. (Hg.): Seiteneinsteiger. Unkonventionelle Politiker-Karrieren in der Parteiendemokratie, Wiesbaden, 2009, 31-60. 
entscheidende Zäsur in der sozialdemokratischen Geschichte, in welcher sie ihren industriegesellschaftlichen Wurzelgrund verlor und ihren sozialetatistischen Planungsanspruch in Richtung einer reformistischen Umgestaltung von Macht und Eigentumsverhältnissen abzulegen anfing, um zehn Jahre vorzulegen.

1973 begann die Welt der alten sozialdemokratischen Arbeiterbewegung unterzugehen. ${ }^{3}$ Das glückliche Vierteljahrhundert der Nachkriegszeit ging zu Ende. Die Zeit „,nach dem Boom“, wie Historiker gern charakterisieren, setzte ein. ${ }^{4}$ Das wirtschaftliche Wachstum verlor an Stabilität und Tempo. Die Massenarbeitslosigkeit, von den Keynesianern in der SPD rhetorisch bereits als Derivat einer überwundenen Zeit angesehen, kehrte zurück. Im Transformationsprozess von der Industrie- zur Dienstleistungsgesellschaft hatten die alten Hochburgen der Sozialdemokratie in den Industrierevieren im Westen und Norden der Bundesrepublik erheblich an Bedeutung eingebüßt. Die Zechen verschwanden, Hochöfen und Werften wurden stillgelegt. So wurden aus den Zentren des Fortschritts, die zugleich rund hundert Jahre lang zentrale Orte einer sozialdemokratischen Assoziationsintensität bildeten, Stätten der sozialen Nachhut, der kulturellen Auflösung zuvor verdichteter sozialmoralischer Milieus. Die Prosperitätsschwerpunkte der Republik verlagerten sich, wechselten in den Süden und Südwesten des Landes, wo die sozialdemokratische Kollektivität sich historisch nicht im größeren Umfang hatte festsetzen können.

Kurz: In diesen frühen 1970er Jahren trat der „Malocher“, jener in der sozialistischen Ikonographie visuell veredelte athletische Industriearbeiter mit kräftigen Muskeln und verlässlicher gewerkschaftlich-sozialistischer Gesinnung, ab sukzessive zunächst, aber eben doch unaufhaltsam. ${ }^{5}$ Die Arbeiterklasse spaltete sich auf und spreizt sich seither weit auseinander: einerseits in die Gewinner, die die zuvor in Gang gesetzten Bildungsreformen beherzt nutzten und Aufstiegschancen, besonders innerhalb des Öffentlichen Dienstes, wahrnahmen; andererseits in die neuen Verlierer, die an den Bildungsanstrengungen nicht teilnahmen oder daran scheiterten, auf Grund geringer beruflicher Qualifikation jedenfalls

3 Vgl. hierzu wie im Folgenden: Walter, F.: Vorwärts oder abwärts? Zur Transformation der Sozialdemokratie, Berlin, 2010; Butzlaff, F./Micus, M./Walter, F. (Hg.): Genossen in der Krise? Europas Sozialdemokratie auf dem Prüfstand, Göttingen, 2011.

4 Vgl. etwa Doering-Manteuffel, A.: Nach dem Boom. Brüche und Kontinuitäten der Industriemoderne seit 1970, in: Vierteljahrshefte für Zeitgeschichte, 55/4 (2007), 567ff.; Ders./Raphael, L.: Nach dem Boom, Perspektiven auf die Zeitgeschichte seit 1970, Göttingen, 2008; auch Jarausch, K.H. (Hg.): Das Ende der Zuversicht? Die siebziger Jahre als Geschichte, Göttingen, 2008.

5 Vgl. ebenfalls Hinrichs, $W$. u.a.: Der lange Abschied vom Malocher. Sozialer Umbruch und die Rolle der Betriebsräte von 1960 bis in die neunziger Jahre, Essen, 2000. 
zunehmend weniger gebraucht wurden. Die aufgestiegenen Gewinner ließen im weiteren biografischen Prozess die zurückfallenden Verlierer in den Arbeiterquartieren allein zurück, strukturierten die Lebenswelt dort nicht mehr, vermittelten keine normativen Orientierungen, stifteten somit weder politischen Sinn noch organisatorischen Halt. Arbeiterquartiere mit einer einst vitalen Solidaritätskultur transferierten sich mehr und mehr zu verwahrlosten Revieren dauerhafter Arbeitslosigkeit, in denen Apathie und negative Individualisierung an die Stelle von Selbstbewusstsein und demonstrativ herausgestellter Gruppenzugehörigkeit traten. Die sozialdemokratische Klassenbasis zerbrach auf diese Weise; die zwei Stränge, die sich aus dem sozialen Schisma entwickelten, schieden sich kräftig. Am Ende waren die lebensweltlichen Gemeinsamkeiten von früher verflogen. ${ }^{6}$

Der neue Prozess der Enthomogenisierung der bundesdeutschen Arbeitnehmergesellschaft, die noch in den 1960er Jahren in vielerlei Hinsicht (versicherungsrechtlich, materiell und sozialkulturell) stärker an Kohärenz gewonnen hatte als je zuvor in der modernen Geschichte Deutschlands, ${ }^{7}$ setzte sich im Verlauf der 1970er Jahre fort. Die Folgen trafen die Sozialdemokraten, welche im vorangegangenen Jahrzehnt noch die im Trend marschierenden Nutznießer der Modernisierungsschübe zu sein schienen, zunächst härter als die anderen Parteien. Denn ihre Anhängerschaft zergliederte sich im Fortgang der Dekomposition überlieferter Vergemeinschaftungen und infolge von neuen Mobilitätsgelegenheiten besonders stark. Nirgendwo sonst fielen die politischen Optionen, Lebenslagen und Einstellungen so different, ja gegenläufig aus wie nun im Potential des sozialdemokratischen Elektorats. Der Terminus, der die sozialdemokratische Problematik der 1970er und insbesondere der 1980er Jahre auf den Begriff bringen sollte, war der des ,Spagats“. ${ }^{8}$ Denn diese Übung einer weit gefächerten, oft widersprüchlichen Wähleransprache hatten Sozialdemokraten zu leisten, wenn es in die Wahlkämpfe ging, um alte oder neue Unterschichten zu halten, marktorientierte Mittelschichten hinzuzugewinnen oder um ökopazifistische Akademiker nicht zu verprellen.

Mit dieser Herausforderung hatten die Sozialdemokraten zu tun, nicht nur in Deutschland, sondern auch in den meisten anderen nord- und westeuropäischen

6 Hierzu auch Walter, F.: Die starken Arme legen keine Räder mehr still, in: Klatt, J./ders.: Entbehrliche der Bürgergesellschaft?, Bielefeld, 2011, $7 \mathrm{ff}$.

7 Vgl. Mooser, J.: Arbeiterleben in Deutschland 1900-1970, Frankfurt a. M., 1984, 224ff.

8 Siehe auch Egle, C./Henkes, C.: Später Sieg der Modernisierer über die Traditionalisten?, in: Egle, C./Ostheim, T./Zohlnhöfer, R. (Hg.): Das rot-grüne Projekt, Wiesbaden, 2003, 67-92, hier 72; Lösche, P.: Ende der Volksparteien, in: Aus Politik und Zeitgeschichte, 51 (2009). 
Ländern. Die gesellschaftlichen Modernisierungswellen hatten nicht nur für den Bruch der sozialdemokratischen Klassenfundamente gesorgt, sie erschwerten auch die Integration der neuen Jugendprotestkultur im vermeintlich ,roten Jahrzehnt $^{\star 99}$ der 1970er Jahre. In diesem Jahrzehnt kristallisierten sich bekanntlich neue Themen und soziale Bewegungen in der nachwachsenden Generation insbesondere der 1950er-Geburtsjahrgänge - heraus, die sich nicht mehr zuvörderst am traditionellen Arbeit-Kapital-Gegensatz entlang politisch deuteten. Nicht zuletzt an der Frage der Kernenergiepolitik des zweiten sozialdemokratischen Bundeskanzlers Helmut Schmidt entfaltete sich eine biografisch dauerhaft enttäuschte Liebe dieser Kohorte zur SPD, die zu einer neuen politischen Repräsentanz - eben den Grünen - führte.

Nun mochten sich die Sozialdemokraten damit in einer paradoxen Dialektik und gewiss nicht mit Absicht einen linksbürgerlichen Koalitionspartner geschaffen haben, der das gegnerische altbürgerliche Lager kräftig dezimierte und so der SPD, im Unterschied zu den schwierigen ersten beiden Jahrzehnten der Bundesrepublik, einen koalitionspolitischen Terraingewinn verschaffte. Schwerer indessen taten sie sich mit der Nachfolgegeneration, den Geburtsjahrgängen der späten 1960er bis frühen 1980er Jahre. Diese Generation hatte den sozialdemokratisch durchwirkten Sozial- oder Wohlfahrtsstaat in den Jahren ihrer primären politischen Sozialisation schon als brüchig und reparaturbedürftig erfahren. Ein Großteil der Zugehörigen dieser Jahrgänge, vor allem wenn sie im global ausgerichteten Privatsektor der Ökonomie tätig waren, neigte während der 1980er und 1990er Jahre - wieder: fast überall in den europäischen Demokratie - den MitteRechts-Parteien zu. Erst die Finanzkrise hat einige aus diesem Spektrum fortbewegt, allerdings im Wesentlichen zu den Grünen, kaum zu den Sozialdemokraten.

\section{Der SPD gelingt der Spagat}

Keiner dieser Belastungsfaktoren - die Erosion des klassischen Milieus, die Desintegration der Wählersegmente - verschwand 1998 gleichsam über Nacht. Und doch gewann die SPD damals nach 16 Jahren der Opposition die Bundestagswahlen. Das lag keineswegs nur am „Medienfaktor Schröder“, wie man gerade unter Journalisten gern kolportierte und sich im Lager der Union bevor-

9 Siehe Koenen, G.: Das rote Jahrzehnt. Unsere kleine deutsche Kulturrevolution 1967-1977, Köln 2001; Faulenbach, B.: Die Siebzigerjahre. Ein sozialdemokratisches Jahrzehnt?, in: Archiv für Sozialgeschichte, XLIV (2004), 1-37. 
zugt über die eigene bittere Niederlage hinweg zu trösten versuchte. Wichtiger war anderes: Im Zentrum der Republik standen zum Ende des 20. Jahrhunderts die in den 1940er, 1950er und 1960er Jahren Geborenen, welche 1998 die tragende Schicht der Eltern und Berufstätigen in der bundesdeutschen Gesellschaft konstituierten. Sie bildeten im Vergleich zu den Generationen davor, die durch die Republik von Weimar, den Nationalsozialismus, den Wiederaufbau unter Adenauer sozialisiert worden waren, tatsächlich und nicht nur in der rhetorischen Einverleibung der Sozialdemokraten eine ,neue Mitte““. ${ }^{10}$ Denn diese neue Mitte war in großen Teilen geprägt von der Ära der ersten Bildungsexpansion, von der Ausdehnung des Wohlfahrtsstaates und vom Ausbau des öffentlichen Sektors, besonders in den Humandienstleistungsbereichen. Sie war geprägt oder vorgeformt von den Aufwallungen von 1968, von den hiervon ausgehenden Emanzipations- und Partizipationsansprüchen, von der Popkultur und der Umstülpung der Wertehierarchien, in ihren jüngeren Kohorten auch und vor allem von den sozialen Bewegungen und den in Massendemonstrationen artikulierten Konflikten der späten 1970er und frühen 1980er Jahre. Das kam nicht in jedem Falle der SPD zugute, dafür oft den Grünen, die als Koalitionspartner der Sozialdemokraten aber schon ungeduldig bereitstanden.

Jedenfalls kamen in der neuen Generationen-Mitte Rhetorik, Sprachbilder und Habitus von Helmut Kohl, der wie zuvor Konrad Adenauer Maß, Mitte und Mehrheit lange Zeit keineswegs erfolglos für sich und seine Union reklamierte hatte, nicht mehr recht an; die „Mittigkeit“ hatte sich kulturell verändert, was ganz erheblich zu den Stimmeneinbußen der Union nicht erst im Jahr 1998 beigetragen hatte. In der nachgewachsenen Generation, in der neu formierten Mitte - vor allem der Jahrgänge 1950 bis 1967 - existierten für Sozialdemokraten und Grüne bereits seit der ersten Hälfte der 1980er Jahre und im Grunde bis in die Gegenwart hinein verblüffend konstante Mehrheiten. Nirgendwo erzielte das altbürgerliche Lager eine so schwache Resonanz wie in der zahlenmäßig üppig besetzten Altersgruppe der letzten baby boomer der Republik. In dieser gewissermaßen sozial-liberal-ökologisch auftretenden neuen Mitte der Gesellschaft

10 Vgl. auch Schwarzkopf, S.: Die „Neue Mitte“. Oder: Wahlkampf als Produkteinführung. Die Bedeutung der Begriffsarbeit für den sozialdemokratischen Machtwechsel in Deutschland 1998, in: Gries, R./Schmale, W. (Hg.): Kultur der Propaganda, Bochum, 2005, 211-250; Dürr, T.: Was ist und wem gehört die „Neue Mitte“? Überlegungen zum Parteienstreit um einen strategischen Begriff, in: Mayer, T./Meyer-Walser, R.C. (Hg.): Der Kampf um die politische Mitte. Politische Kultur und Parteiensystem seit 1998, München, 2002, 20-42. 
hatte sich das Wahlergebnis von 1998 schon über mehrere Jahre im Vorfeld aufgebaut, zuerst in den Ländern und Kommunen, dann schließlich im Bund.

Und auch die sozialdemokratische Doppelspitze des Wahlkampfes 1998 aus Oskar Lafontaine und Gerhard Schröder bot ein plausibles Konzept, um das sozialdemokratische Dilemma des Spagats zu lösen. ${ }^{11}$ Die SPD hatte Tradition zu wahren, durfte sich aber auch von der Moderne nicht abschotten; sie brauchte die Reste ihres Milieus, durfte sich aber nicht darauf beschränken; sie musste ihre Kernschichten motivieren, durfte aber das Rochadesegment zum bürgerlichen Lager nicht abschrecken. Lafontaine hielt die sozialdemokratischen Traditionsschichten bei Laune, indem er die sozialen Ungleichgewichte in der Republik anprangerte und dem Neoliberalismus den Fehdehandschuh vor die Füße warf. Schröder hingegen richtete die Ansprache stärker an die Grenzwähler im Zwischenfeld von Union und SPD, die sich nach 16 Jahren Kohl einen wie auch immer gearteten neuen gesellschaftlichen und ökonomischen Schwung erhofften. Auf diese Weise spiegelte die Doppelspitze der SPD die ambivalente nationale Befindlichkeit der Bevölkerungsmajorität in der zweiten Hälfte der neunziger Jahre wider. Denn auch die Mehrheit der Deutschen sprach sich im Prinzip für „Innovationen“ aus, war jedoch verängstigt, sobald konkrete Vorschläge in diese Richtung gingen. Die SPD-Doppelspitze deckte Zuversicht und Ängstlichkeit ab, stellte Reform wie Antireform in Aussicht. Sie bot auf diese Weise ein Plateau, auf dem sich Wächter des Wohlfahrtsstaates und Propheten der Deregulierung, ergraute Gewerkschaftsfunktionäre und juvenile Firmengründer elektoral ansiedeln konnten.

Allerdings ging daher von der Bundestagswahl, trotz des eindeutigen Resultats zugunsten von Rot-Grün, kein eindeutiges Signal aus, keine scharf vorgezeichnete Willensrichtung des demos. Dadurch unterschied sich der Regierungswechsel 1998 deutlich von den Wenden der Jahre 1969 und 1982/83, als sehr viel klarer war, wohin die Gesellschaft mehrheitlich strebte. Nochmals: 1998 erhoffte sich der eine, wahrscheinlich eher geringe Teil der sozialdemokratischen Wählerschaft neuen Impetus für marktwirtschaftliche Reformen und kraftvolle Deregulierungsinitiativen. Der andere, wohl größere Teil wehrte sich genau dagegen und erwartete vom neuen Bonner Kabinett zuallererst Schutz, Sicherheit und

11 Vgl. auch Bergmann, K.: Der Bundestagswahlkampf 1998. Vorgeschichte, Strategien, Ergebnisse, Wiesbaden, 2002, 18ff.; auch Merkel, $W$. u.a.: Die Reformfähigkeit der Sozialdemokratie. Herausforderungen und Bilanz der Regierungspolitik in Westeuropa, Wiesbaden, 2006, 160f; Walter, F.: Vom Betriebsrat der Nation zum Kanzlerwahlverein? Die SPD, in: Pickel, G./Walz, D./Brunner, W. (Hg.): Deutschland nach den Wahlen, Opladen, 1999, $227 \mathrm{ff}$. 
Zuwendung. Wohin auch immer die Regierung also ging, sie musste einen Teil ihrer heterogenen Wählerkoalition vor den Kopf stoßen.

\section{Der Massenexodus der Wähler}

Und sie marschierte 2003, nach zahlreichen begründungslosen Zickzackwendungen, auf die Agenda 2010 zu. Die Vorarbeiten dazu hatte ein kleiner Zirkel unter der Regie des Kanzleramtschefs Frank-Walter Steinmeier bereits Ende 2002 geleistet - ansatzlos, zumindest nicht in einem nachvollziehbaren Nexus zum Wahlprogramm von 1998 oder den Wahlkampfslogans von 2002. „So geriet die Agenda 2010 zu einer klassisch exekutiven Strategie eines Stoßtrupps, um sich aus höchster Not mit einem Befreiungsschlag zu retten und die Initiative wieder an sich zu reißen."12 Öffentlich gemacht wurde die neue Marschroute Mitte März 2003 durch eine Regierungserklärung Gerhard Schröders. Ein stringent durchkomponiertes und im Detail ausformuliertes Konzept war die „Agenda 2010“ zu keinem Zeitpunkt. Aber mit dem Vortrag Schröders im Bundestag lagen die konstitutiven Zielkategorien der rot-grünen Bundesregierung für die folgenden zwei Jahre fest: aktivierender Sozialstaat, Fordern und Fördern, Selbstbeteiligung und Eigenverantwortung, Investition statt Konsumtion, Zukunft statt Vergangenheit.

Es waren keineswegs Phantomschmerzen, welche die Ursache dafür bildeten, dass im weiteren Fortgang sozialdemokratischer Regierungsbeteiligung Hunderttausende von Mitgliedern und Millionen von Wählern der Sozialdemokratie enttäuscht den Rücken zuwandten. Denn niemals zuvor im 20. Jahrhundert hatte sich in einer solchen Geschwindigkeit die soziale Ungleichheit, also der Wohlstandsgraben zwischen den Einkommensverhältnissen oben und unten, so stark vertieft wie in den sozialdemokratischen Regierungsjahren seit 1999. Die Armutsquote war ab 2000 von 12 auf 18 Prozent hochgeschnellt. ${ }^{13}$ Die Zahl derjenigen, die man gewöhnlich als working poor bezeichnet, hatte sich in den Schröder-Jahren verdoppelt. Die neuen Jobs, welche den Agenda-Reformen seither gutgeschrieben werden, bestanden in der Hauptsache aus Mini-Jobs, unfreiwilligen Teilzeit- und Kurzarbeitsplätzen. Etliche Hundertausende, die auf diese Weise unsicher beschäftigt waren, lebten zugleich in Teilen von Hartz IV, weil die Entlohnung für ihre Erwerbstätigkeit zum Leben nicht reichte. Und die

12 Strünck, C.: Die SPD unter Kurt Beck, in: Baus, R.T. (Hg.): Zur Zukunft der Volksparteien, St. Augustin/Berlin, 2009, 33-44, hier 37.

13 Akribisch hierzu und im Folgenden Lohauß, P.: Die Rückkehr der Klassengesellschaft, in: Kommune, 27/5 (2009), 6ff. 
Hartz IV-Administration hatte - so zumindest der frühere christdemokratische Bundesminister für Arbeit und Sozialordnung Norbert Blüm - „den Sozialstaat zum Überwachungsstaat ${ }^{\text {'14 }}$ gemacht. Einen größeren Vertrauenskredit gewährte die rot-grüne Bundesregierung dagegen den Betreibern von Hedgefonds, denen sie durch das Investmentgesetz vom 15. Dezember 2003 noch mehr Raum zur freien Entfaltung gab. Das setzte sich auch in der Großen Koalition mit der CDU/CSU fort. 2006 riet Jörg Asmussen, enger Vertrauter von Hans Eichel und später in der Regierung der Großen Koalition Staatssekretär im Finanzministerium, den Banken bei Investitionen in ABS-Produkte „keine unnötigen Prüfungsund Dokumentationspflichten“ aufzuerlegen. ${ }^{15}$ Und mit Hartz IV, mit der Zusammenfügung von Sozialhilfe und Arbeitslosenhilfe auf dem Niveau der ersteren, hat die sozialdemokratisch geführte Bundesregierung die umfangreichste Leistungskürzung in der bundesdeutschen Sozialgeschichte vollzogen. ${ }^{16}$ Als Schröders Kanzlerschaft ablief, stand die Arbeitnehmerschaft hierzulande materiell und rechtlich deutlich schlechter da als im Jahr der rot-grünen Inthronisierung. Klaus-Peter Schöppner von TNS-Emnid präsentierte den Befund, dass 1998, nach 16 Jahren Kohl'scher Kanzlerschaft, 55 Prozent der Bürger die deutsche Gesellschaft als ungerecht qualifiziert hatten; nach sieben Jahren von RotGrün - ursprünglich angetreten, um die soziale Asymmetrie zu korrigieren - war die Quote auf 78 Prozent angestiegen. ${ }^{17}$

Die deutschen Sozialdemokraten verloren in ihrer Regierungszeit Wähler in einem Ausmaß, das singular in der bundesdeutschen Geschichte steht. Beinahe die Hälfte der gewerkschaftlich organisierten Arbeiter - über hundert Jahre die Avantgarde, die Kader des proletarischen Vorfelds der SPD - entfernte sich aus dem sozialdemokratischen Elektorat. 1998 hatten sie noch zu 60 Prozent die SPD gewählt. Elf Jahre später belief sich der Anteil nur noch auf 35 Prozent. Ein Minus von 16 Prozentpunkten war in dieser Gruppe allein in den vier Jahren der Großen Koalition zu verzeichnen. Bei den jungen Bundesdeutschen im Berufseintrittsalter gelang es gar der FDP zum Ende des letzten Jahrzehnts, die SPD hinter sich zu lassen. Im erwerbstätigen Teil der Bevölkerung hat in diesem Jahr-

14 Interview mit Norbert Blüm: „Hartz ist Pfusch“, in: süddeutsche.de, 13.09.2009.

$15 \mathrm{Vgl}$. Asmussen, J.: Verbriefung aus Sicht des Bundesfinanzministeriums, in: Zeitschrift für das gesamte Kreditwesen, 19 (2006), 11.

16 Vgl. Trampusch, C.: Sozialpolitik. Vorwärts- und Rückwärtsreformen und Neuvermessung von Solidarität, in: WSI-Mitteilungen, 7 (2008), 365-371.

17 Vgl. Schöppner, K.-P.: „Wirtschaftspolitik“ ist wahlschädlich, in: Cicero 9 (2007); etwas anders die Zahlen des Autors in Schöppner, K.-P.: Therapie für die Sozialdemokratie, in: Neue Gesellschaft/Frankfurter Hefte, 11 (2008), 44. 
zehnt eine veritable Massenabwanderung von der SPD stattgefunden. Statt 16.194.665 Wahlbürger im Jahr 2005 gaben bei den Bundestagswahlen 2009 nur noch 9.990.488 Bundesdeutsche den Sozialdemokraten ihre Stimme. ${ }^{18}$ Allein die Rentner hielten die SPD 2009 noch über der 20-Prozent-Marke. Insgesamt war der Zustimmungsanteil für die Sozialdemokraten bei allen Wahlberechtigten von 41,4 Prozent im Jahr 1972 auf 16,1 Prozent geschrumpft.

\section{IV. „Vermittelschichtung“ einer Arbeiterpartei}

Zugleich versiegten die Mitgliederquellen (vgl. Abb. 1). Begonnen hat dieser Prozess indes schon in den Jahren der Opposition während Kohls Kanzlerschaft. Seit 1990 hat die SPD Mitglieder im Umfang von viereinhalb Großstädten eingebüßt. Die alte Massenpartei, deren Stolz lange die kollektiven Großbataillone vorzüglich in Bewegung zu setzender Aktivisten waren, hat seit 1990 nahezu 450.000 Mitglieder verloren, ist 2008 unter den Bestand der früheren christdemokratischen Honoratiorenorganisation gerutscht - ein historisch tiefer Einschnitt in der Geschichte politischer Organisationskulturen. Zum Jahreswechsel 2010/2011 meldete die CDU 505.314 Mitglieder; die Sozialdemokraten wiesen mit 502.062 gut 3.000 Mitglieder weniger aus. ${ }^{19}$ In den Monaten darauf unterschritten beide „Volksparteien“ die 500.000-Mitglieder-Marke. In den sozialliberalen Regierungszeiten besaß noch nahezu jeder sechzigste Bundesbürger ein SPD-Parteibuch, zum Ende des ersten Jahrzehnts im 21. Jahrhundert entrichtete nur jeder Zweihundertste regelmäßig Beiträge für seine sozialdemokratische Parteizugehörigkeit. ${ }^{20}$ Am stärksten fielen die Verluste der SPD bezeichnenderweise in ihren früheren industriellen Kernregionen und Hochburgen aus: im Saarland, in Nordrhein-Westfalen, in Bremen und in Hamburg. Im Vergleich zum oft verklärten goldenen sozialdemokratischen Jahrzehnt, den 1970er Jahren, ist dort der Mitgliederbestand auf knapp ein Drittel zusammengeschmolzen. ${ }^{21}$ Sollte sich der Mitgliederschwund in der SPD der letzten beiden Jahrzehnte in der Zukunft linear fortsetzen, dann würde der letzte Sozialdemokrat etwa im Jahr 2041 die Lichter im Willy-Brandt-Haus ausschalten. ${ }^{22}$

$18 \mathrm{Vgl} . \mathrm{Neu}, \mathrm{V}$ : Bundestagswahl in Deutschland am 27. September 2009. Wahlanalyse, hg. von der Konrad-Adenauer-Stiftung, Berlin, Dezember 2009.

19 Vgl. „Die Krise in der SPD“, in: die tageszeitung, 20.12.2010; CDU und FDP laufen die Mitglieder davon, Focus Online, 15.6. 2011.

20 Siehe Nachtwey, O.: In der Mitte gähnt der Abgrund, in: Blätter für deutsche und internationale Politik, 8 (2008), $55 \mathrm{ff}$.

21 Hierzu Walter, F.: Die SPD. Biographie einer Partei, Reinbek bei Hamburg, 2009, $277 \mathrm{ff}$.

22 Siehe Wiesendahl, E.: Volksparteien. Aufstieg, Krise, Zukunft, Opladen, 2011, $142 \mathrm{ff}$. 
Abbildung 1: Mitgliederentwicklung der SPD 1906-2011

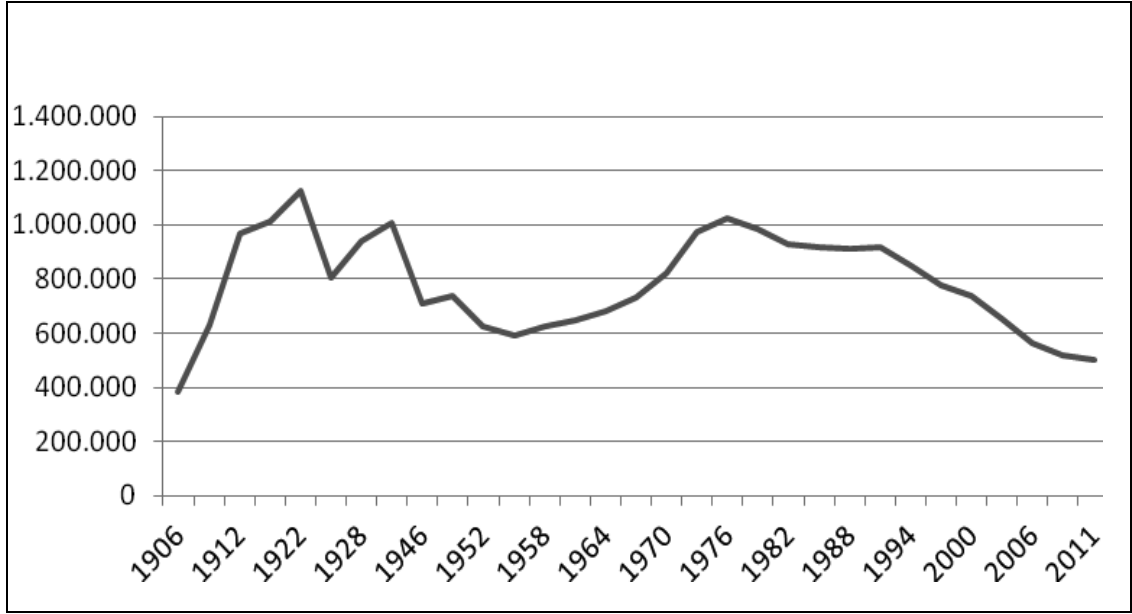

Quelle: Eigene Darstellung.

Überdies: Fast die Hälfte aller sozialdemokratischen Mitglieder (ca. 47 Prozent) ist mittlerweile über sechzig Jahre alt, das Durchschnittsalter der Sozialdemokraten liegt 2011 bei 58 Jahren (zum Vergleich: bei den Grünen bei 46, den Freien Demokraten bei 51 und der CDU/CSU ebenfalls bei 58 Jahren), nicht einmal zehn Prozent sind in einem Alter von unter 36 Jahren. Sozialstrukturell dominieren in der SPD mittlerweile die Rentner und Pensionäre, gefolgt von den Angestellten. Der Anteil der Arbeiter liegt in der Partei des früheren Industrieproletariats nur noch bei acht Prozent, sechs Prozent unter der Arbeiterquote in der Gesamtbevölkerung - auch das ein Wendepunkt in der Geschichte der ,sozialen Demokratie“ hierzulande. Die stärkste Gruppe unter den aktiv erwerbstätigen Mitgliedern bilden die Beamten mit 23 Prozent. An der Spitze der Sozialstruktur stehen indessen die Rentner mit 34 Prozent. ${ }^{23}$

Im Jahr 2007 ergab ein Befund der Organisationsabteilung in der SPD-Zentrale, dass in rund zwanzig Prozent der Ortsvereine seit fünf Jahren kein einziges Neumitglied mehr aufgenommen worden war. ${ }^{24}$ Ähnlich ernüchternd fiel eine Mitgliederbefragung des Instituts polis + sinus im Auftrag des Willy-BrandtHauses im Frühjahr 2010 aus. ${ }^{25}$ Der neue Parteichef Sigmar Gabriel äußerte

23 Siehe Mitgliederzusammensetzung der SPD auf: http://www.bpb.de/files/M1C6P3.pdf.

24 Vgl. auch Strünck, C.: Die SPD unter Kurt Beck, a.a.O., 35.

25 Vgl. hierzu und im Folgenden SPD: Ergebnisse einer bundesweiten Befragung der SPD-Ortsvereine, Berlin, 2010. 
danach Zweifel an der Kampagnenfähigkeit seiner Partei, die in Gefahr gerate, zu einem closed shop zu verkommen. ${ }^{26}$ Die Zahl der Ortsvereine war in den vergangenen Jahren erheblich zurückgegangen. In den verbliebenen lokalen Sektionen fanden offensive, nach außen gewandte Aktivitäten kaum noch statt. Die Fäden zur programmatisch häufig zitierten Zivilgesellschaft waren denkbar dünn. Regelmäßige Kooperationen mit Umweltinitiativen reklamierten lediglich sieben Prozent der sozialdemokratischen Ortsgruppen für sich. Marginal fiel auch die stetige Zusammenarbeit mit den Gewerkschaften aus, da ein solches Beziehungsgeflecht zwischen den beiden früher tragenden Säulen der Arbeiterbewegung nur in acht Prozent der SPD-Ortsvereine zu finden war. In über der Hälfte der Orte, die sich an der Mitgliederbefragung beteiligten, existierte keine innerparteiliche Arbeitsgemeinschaft. Überdies brachte die Expertise hervor, dass das Gros der Sozialdemokraten den politischen Kampf für „gerechte Löhne“ sowie für wirksame Regeln der Finanzmärkte als besonders wichtig taxierte, dagegen der „Gleichstellung von Lebenspartnerschaften“, der „Integration von Einwanderern“ oder der „demokratischen Mitwirkung der Bürger in allen Lebensbereichen" einen bemerkenswert geringen Rang für die Arbeit der Gesamtpartei beima $\beta$.

Das Nachrichtenmagazin Der Spiegel schrieb mokant von der SPD als „Schrumpf-Partei Deutschland“. ${ }^{27}$ Doch bei solcherlei Spöttereien wird in der Regel übersehen, dass der drastische Mitglieder- und Organisationsverlust, mit dem die Sozialdemokraten konfrontiert sind, auf eine Entwicklung zurückgeht, die unzweifelhaft auch positive Seiten aufweist. Denn: Die Sozialdemokraten sind Opfer des eigenen Erfolgs geworden. Die Sozialdemokratie ist im Zuge des Aufstiegs der klassischen Facharbeiterelite zur Partei einer neuen gesellschaftlichen Mitte geworden. In einer jüngeren Mitgliederbefragung ordnen sich nicht weniger als 96 Prozent der sozialdemokratischen Mitglieder den Mittelschichten zu; nur drei Prozent siedeln sich selbst in der Unterschicht an. ${ }^{28}$ Die Zugehörigen der mittleren und oberen Mittelschicht - 74 Prozent der organisierten Sozialdemokraten reihen sich hier ein - sind oft vergleichsweise ressourcenstarke Menschen mit akademischen Abschlüssen, benötigen nicht mehr unabdingbar das Kampfgeschirr der disziplinierten Organisation, sind nicht mehr angewiesen auf den Paternalismus von Partei- oder Gewerkschaftsfunktionären. Die SPD hat

26 Zitiert nach: „Viele Ortsvereine ohne politische Aktivität“, in: FAZ, 01.06.2010; vgl. auch Strohschneider, T.: Die Schwäche des Gegners, in: Der Freitag, 10.6.2010.

27 Vgl. Dramatischer Schwund. SPD bald nicht mehr größte Volkspartei, in: Spiegel Online, 03.02.2008.

28 Vgl. die Mitgliederzusammensetzung der SPD auf: http:/www.bpb.de/files/M1C6P3.pdf. 
sich selbst als Honoratiorenpartei sozialstaatlich protegierter Aufsteiger gesellschaftlich nach oben katapultiert. Ihre Kerngruppe - Lehrer, Dezernenten, Referatsleiter etc. - verfügt individuell über Ressourcen und Kompetenzen, die den früheren empfundenen Zwang zur Kollektivität und Organisationsbindung entbehrlich machen.

Im Zuge dieses Prozesses blieb die SPD nicht mehr genuine Partei der Arbeiter. Gut einhundert Jahre war sie stolz darauf, eben dies gewesen zu sein. Sie hatte ein Großteil ihres Selbstbewusstseins daraus gezogen, als politische Repräsentantin jener sozialen Formation zu agieren, welche die ökonomischen Werte schuf, mithin produktiv war - im Gegensatz etwa zu dem von Georg Grosz als parasitärer, zigarrenrauchender Couponschneider karikierten Kapitalisten der 1920er Jahre. In den neuen organisationsentbundenen Unterschichtquartieren der Republik traten der SPD allerdings vorwiegend Opfer, Verlorene, Ausgemusterte, Marginalisierte entgegen, die nicht einmal für die „ökonomische Ausbeutung“ einen Nutzen besaßen. Als Anwärter für Zukunftsprojekte und kraftvolle Wirtschaftsreformen waren sie nicht ernsthaft ins Kalkül zu ziehen. Eine Klasse der Zukunft, eine Akteurin der sozialen Befreiung verkörperte die verbliebene Unterschicht gewiss nicht mehr, wie es die Theoretiker des Sozialismus in früheren Zeiten erhofft hatten.

So trennten sich das soziale Unten und die Sozialdemokraten voneinander, mehr in einem schleichenden Prozess als in einem bewusst vollzogenen Akt. Ohne Probleme lief das für viele Sozialdemokraten alter Couleur nicht ab. Sie taten noch eine ganz Zeit so, als könne ihre Partei irgendwie doch noch die Partei der Arbeiter, zumindest die der „kleinen Leute“ bleiben.

Perdu sind unterdessen ebenfalls die in den Aufstiegsjahrzehnten des Sozialismus so vielfältigen Intellektuellen und Parteitheoretiker, deren Bogen sich von Karl Marx und Friedrich Engels über Eduard Bernstein, Karl Kautsky, Rosa Luxemburg bis hin zu Rudolf Hilferding, Alexander Schifrin, Theodor Dan, Georg Decker, Eduard Heimann, Siegfried Marck, Hermann Heller, Gustav Radbruch, später dann Willi Eichler, Peter von Oertzen, Richard Löwenthal - um nur einige unter vielen weiteren aufzuzählen - spannte. ${ }^{29}$ Der Verlust an Intellektualität und Begründungsfähigkeit hat in der Generation nach Willy Brandt und Erhard Eppler unzweifelhaft eine geistig-politische Ortlosigkeit geschaffen. Auch deshalb büßten die Sozialdemokraten während der letzten dreißig Jahre die

29 Vgl. Walter, F.: „Moralanwälte“ und „Störungsfaktoren“. Die Sozialdemokratie und ihre Intellektuellen, in: Indes. Zeitschrift für Politik und Gesellschaft, Herbst 2011, 30ff. 
Vorstellung von ihrem geschichtlichen Lebensinhalt ein, so dass sie sich stattdessen wechselhaft und zum Teil bemerkenswert widersprüchlich politisch ausdrückten. Und sie liehen sich, gerade im ersten Jahrzehnt des 21. Jahrhunderts, programmatische Versatzstücke aus verschiedensten fremden Depots, da ein eigener Vorrat kaum noch bestand. Geistig ist der „demokratische Sozialismus“ der Sozialdemokraten, wie ein früheres Mitglieder der SPD-Orientierungskommission bitter bilanzierte, nur noch „kalte Asche““ ${ }^{30}$ Lediglich exaltierte Randgruppen erhöhen die Arbeiterklasse unverdrossen zum „historischen Subjekt" geschichtswendender Kraft. Kaum jemand vertraut weiterhin und trotz zuletzt gewachsener Marktskepsis auf mächtige sozialistische Staatsorgane, welche Produktion, Verteilung und Konsumtion planvoll zu steuern vermögen.

Gründlich entkoppelt haben sich inzwischen des Weiteren die Lebenswelten von Sozialdemokraten und Gewerkschaftern. Beide Sphären haben lange das Dach einer gemeinsamen Arbeiterbewegung getragen, in der sich Betriebserfahrungen und politische Professionalität verknüpften. In den 1950er und 1960er Jahren pflegte die SPD bei Wahlen noch prominente Gewerkschafter auf einen der vordersten drei Plätze der Landesliste zu setzen. ${ }^{31}$ Doch heute gehört kein Gewerkschaftsführer mehr der sozialdemokratischen Bundestagsfraktion an; überhaupt ist der Anteil von Gewerkschaftsmitgliedern in der Mannschaft von FrankWalter Steinmeier gegenüber der Regierungszeit von Helmut Schmidt, als (1980) noch 218 von 238 Parlamentarier Mitglied einer DGB-Gewerkschaft waren, ${ }^{32}$ um gut 25 Prozentpunkte zurückgegangen. Insgesamt sind fast 400.000 der knapp 500.000 SPD-Mitglieder jetzt ohne Gewerkschaftszugehörigkeit - auch hier: ein historischer Tiefpunkt. Dafür haben über achtzig Prozent der SPDParlamentarier eine akademische Qualifikation. Die lokalen Betriebsräte sind nicht mehr zugleich stellvertretende Ortsvereinsvorsitzende und/oder Mitglieder von Stadtratsfraktionen der SPD. Dabei ist den Sozialdemokraten „die Aufkündigung der ,privilegierten Partnerschaft' zwischen Sozialdemokratie und Gewerkschaften nicht gut bekommen, wie desaströse Umfragen vor und die Ergebnisse nach der Bundestagswahl 2009 belegen. “33

30 Vgl. Vilmar, F.: „,...nur noch ein Haufen kalter Asche“. Aufstieg und Zusammenbruch des Sozialismus, in: UTOPIE kreativ, 151 (Mai 2003), 415ff.

31 Vgl. hierzu Lösche, P.: Verbände und Lobbyismus in Deutschland, Stuttgart, 2007, 74

32 Vgl. Wehler, H.-U.: Deutsche Gesellschaftsgeschichte 1949-1990, München, 2008, 11.

33 Urban, H.-J.: Niedergang oder Comeback der Gewerkschaften, in: Aus Politik und Zeitgeschichte, 1314 (2010), 3ff., hier 4. 


\section{Zyklische Erneuerungsversuche}

Es ist in Anbetracht der zahlreichen Indizien des Niedergangs der deutschen Sozialdemokratie, der jüngsten Verlusterfahrungen, Schwundstufen und Krisenzeichen kaum verwunderlich, wenn nach der Niederlage bei der Bundestagswahl 2009 abermals die Reform der Partei auf die Tagesordnung gesetzt wurde. So hat es sich in den letzten Dekaden schließlich stets zugetragen: Jedes Mal - und das bedeutet seit 1990 etwa alle fünf Jahre - war die Reformdebatte Ausfluss frustrierender Wahlresultate und eines tiefgreifenden, demoskopisch ermittelten Vertrauensentzuges, forcierter Mitgliederrückgänge und einer dauerkritischen Medienberichterstattung. Es zeigen sich zwischen der aktuellen Operation am Körper der Partei und den vorangegangenen Eingriffen mithin einige Gemeinsamkeiten, die generelle Schlussfolgerungen über Parteireformen zulassen. Die Stimmen der Erneuerung erheben sich einerseits in Phasen spürbaren Niedergangs. Und die Reformen setzen andererseits immer an der Organisation an, die Lösung der Probleme wird in einer Modifikation der innerparteilichen Strukturen, Arbeitsweisen und Entscheidungsprozeduren gesucht.

Beides ist nicht unproblematisch, der Erneuerungsdiskurs wird gleichermaßen kurzsichtig wie einseitig geführt. Zum einen werden Organisationsreformen, sofern sie als Pawlowscher Reflex auf zyklische Zustimmungskrisen daherkommen, dem generellen Bedarf nach kraftvollen Institutionen nicht gerecht. Auf die Funktionen von Kollektivorganisationen können moderne Gesellschaften generell nicht verzichten, dies gilt - nur scheinbar paradox - umso mehr in Zeiten rapiden Wandels, in denen die bestehenden Organisationsstrukturen regelmäßig als Relikte vergangener Zeiten dargestellt werden. Doch ist gerade dann der Einzelne auf die entlastende Wirkung von Institutionen und Organisationen angewiesen, die Unsicherheiten reduzieren und Entscheidungszwänge abbauen.

Für den Philosophen Norbert Bolz liegt die Entwicklungsgarantie fortschrittlicher Zivilisationen eben darin begründet, dass die Menschen selbst nicht alles wissen und intellektuell durchdringen wollen. „Nicht das, was Menschen denken, sondern das, was man ihnen zu denken erspart, bringt den zivilisatorischen Fortschritt. . ${ }^{\text {34 }}$ Die Bedeutung kraftvoller Organisationen beschränkt sich insofern nicht punktuell auf temporäre (Partei-)Krisen, der Erhalt ihrer Lebendigkeit und Funktionsfähigkeit - auf Parteien gemünzt vor allem: einer erfolgreichen Ver- 
mittlung zwischen Staatshandeln und Bevölkerungsinteressen - ist vielmehr eine Daueraufgabe.

Zum anderen aber haben die Reformen der Vergangenheit technisch allesamt einseitig und ausschließlich auf Organisatorisches abgezielt. Strukturen sollten geöffnet, Beteiligungsverfahren ausgebaut und Kommunikationsmedien modernisiert werden. Der Bundesgeschäftsführer der deutschen SPD in den frühen 1990er Jahren, Karlheinz Blessing, mahnte dagegen schon vor zwanzig Jahren zur Selbstbeschränkung: ,Die Parteiorganisation darf nicht Selbstzweck sein, sie muss Instrument sein, mit und für Menschen diese Gesellschaft zu verändern. “35 Mehr noch: Blessing betonte, dass sich das politische Ziel in der Organisation spiegeln muss. Demzufolge müssten im Vorfeld einer Organisationsreform die langfristigen politischen Fluchtpunkte, Ziele und Visionen diskutiert werden, müsste zunächst das programmatische Fundament gegossen werden, dessen Profil und Zuschnitt sodann die organisatorische Form vorgeben würde.

Diesbezüglich bieten die aktuell in der SPD diskutierten Reformmaßnahmen durchaus Anknüpfungspunkte. Sozialdemokratische Parteien sind historisch die Interessenvertretungen und Repräsentanzen derjenigen, die sich alleine nicht helfen können, die ihre individuelle Machtlosigkeit durch die Macht des Kollektivs ausgleichen müssen und sich darum im Gefüge der sozialdemokratischen Organisation engagieren. Als Vertretungsagentur von Arbeitern gegründet, deren wirtschaftliche Unselbständigkeit in der Industriegesellschaft von Unternehmern ausgenutzt wurde, deren Einzelstimme in den abgestuften Wahlrechten der konstitutionellen Monarchien wenig zählte und deren Aufstiegswege in der Klassengesellschaft des späten 19. und frühen 20. Jahrhunderts blockiert waren, ist die SPD folglich eine traditionelle Mitgliederorganisation und „Kümmererpartei“.

Wenn in der Sozialdemokratie - im Übrigen nicht bloß in Deutschland, sondern ganz ähnlich in einer Vielzahl europäischer Länder - nun von der Notwendigkeit gesprochen wird, die lokalen Parteigliederungen zu stärken, auf die Menschen wieder spürbarer zuzugehen, vor Ort besser sichtbar und in der Öffentlichkeit präsenter zu sein, dann schwingt in der Revitalisierung der Graswurzelarbeit eine Wiederbelebung der oben genannten Kümmererstrategie mit. ${ }^{36}$ Und wenn darüber hinaus über neue Konzepte zur Mitgliederwerbung und Mitgliederbindung

35 Blessing, K.: SPD 2000 - Tradition und Modernisierung, in: Heimann, H. (Hg.): Sozialdemokratische Traditionen und Demokratischer Sozialismus 2000, Köln, 1993, 214-228.

36 Vgl. Butzlaff, F./Micus, M./Walter, F.: Im Spätsommer der Sozialdemokratie?, in: Dies. (Hg.): Genossen in der Krise?, a.a.O., 271-300. 
sinniert wird, wenn die Beteiligungsmöglichkeiten von Mitgliedern wie Sympathisanten erweitert und Impulse zur Öffnung der Partei gesetzt werden sollen, so fügen sich auch diese Reformvorschläge in die Tradition der sozialdemokratischen Massenmitgliederpartei ein, zu der immer auch Aktivität, Partizipation, Engagement gehört haben. Im Übrigen: Die Absicht, eine stärkere Beteiligung der Mitglieder, eine veränderte Elitenrekrutierung und die Öffnung der Partei nach außen zu erreichen, ist auch insofern konsequent und schlüssig, als Umfragen konstant große Mehrheiten für erweiterte Mitspracherechte und direktdemokratische Entscheidungsverfahren ermitteln. ${ }^{37}$

Allerdings birgt Basisdemokratie für Parteien - und insbesondere Volksparteien - auch etliche Tücken. Mitgliederentscheide kollidieren etwa mit innerparteilichen Ausgleichsverfahren und Proporzregeln, die gerade für heterogene Abbilder der gesellschaftlichen Vielfalt, wie sie Volksparteien zu sein beanspruchen, schwer verzichtbar sind. Darüber hinaus legen Basisentscheidungen die sozialdemokratische Führung in ihrem Handeln fest - und reiben sich insofern mit dem alltagspolitischen Erfordernis rascher Antworten und flexibler Reaktionen der Parteispitze auf unplanbare oder jedenfalls unvorhergesehene Entwicklungen. Hinzu kommt, dass die Direktdemokratie die Flügel, Funktionäre und Arbeitsgemeinschaften, kurz: den Mittelbau der SPD zugunsten der Parteispitze entmachtet, die über die Medien direkt in Kontakt mit den einfachen Mitgliedern treten kann. Wobei trotz - oder gerade wegen - formal ermächtigender und gleichberechtigender Mitgliedermitbestimmung auch in basisdemokratischen Parteien Hierarchien nicht nur zwischen Bundesspitze und Ortsebene, sondern auch zwischen besser und schlechter organisierten Interessen bestehen. Gerade Mitgliederentscheide begünstigen jene innerparteilichen Gruppen, die ihre Zugehörigen effektiv mobilisieren und relativ geschlossen zur Stimmabgabe bewegen können.

Irrig ist gleichfalls die Annahme, mit Basisvoten einer aufgeklärteren, abgewogeneren und differenzierteren Politik zum Durchbruch zu verhelfen. Das Gesellschaftsbild der Befürworter von Basis- oder Volksbefragungen ist oft keinesfalls pluralistisch, sondern ganz im Gegenteil populistisch, das heißt eine homogen gedachte Bevölkerung wird abgehobenen Eliten gegenübergestellt. Die Befürwortung von mehr Basispartizipation geht denn auch vielfach problemlos mit Forderungen nach einer kompromisslosen Umsetzung des Mehrheitswillens und überhaupt mit Sympathien für einen „starken Mann“ an der Spitze des Staates 
zusammen. ${ }^{38}$ Vor diesem Hintergrund überrascht schließlich auch die Affinität von populistischen Bewegungen und von Parteien am Rand des politischen Spektrums für direktdemokratische Beteiligungsinstrumente nicht. Ganz abgesehen davon, dass die erweiterten Beteiligungserfordernisse den Einzelnen mit gesteigerten Anforderungen konfrontieren, sich zu informieren, mitzudiskutieren und politische Fragestellungen intellektuell zu durchdringen - und die insofern ganz allgemein die Vermittelschichtung der SPD und speziell die innerparteiliche Dominanz der zeitreichen Beschäftigten des Öffentlichen Dienstes nur noch verstärken dürften. ${ }^{39}$

Auch ob direktdemokratische Beteiligungselemente wirklich zu einem Mehr an Beteiligung führen, ist durchaus unklar. Wo einzig die eigene Stimme abzugeben und ein Kreuz zu machen ist, werden oft außergewöhnlich hohe Mobilisierungsquoten erzielt. Bei der Urwahl des Vorsitzenden der Bundespartei beteiligten sich 1993 über fünfzig Prozent der Mitglieder, in Schleswig-Holstein an der Abstimmung über den Spitzenkandidaten bei der nächsten Landtagswahl im Februar 2011 gar knapp siebzig Prozent. Zum Vergleich: Durch die konventionellen Sitzungen und Versammlungen werden in der Regel nur ca. 10-15 Prozent der Mitglieder erreicht. ${ }^{40}$ Sobald jedoch die Beteiligung aufwendiger und zeitintensiver wird, nimmt sie drastisch ab - weshalb der neue Landesparteivorsitzende in Niedersachsen insgesamt nur von mageren fünf Prozent der Mitglieder bestimmt wurde (hier war die Stimmabgabe nur am Ende von sieben Regionalkonferenzen im Anschluss an eine mehrstündige Kandidatenvorstellung und Diskussion möglich).

Aus vielen dieser Gründe hat die SPD seit Jahren schon bei ihren Zukunftswerkstätten große Mobilisierungsprobleme, obwohl die sich an Mitglieder und NichtMitglieder gleichermaßen wenden. Die viel geforderten Themeninitiativen, die bereits eingerichtet worden sind, leiden in der Regel unter mangelnder Beteiligung bzw. einem zu geringen Interesse. Das Gros der SPD-Mitglieder ist weitgehend deaktiviert, fordert laut der letzten SPD-Mitgliederbefragung zwar allgemein mehr Beteiligung und Mitbestimmung, empfindet ihr eigenes geringes Engagement aber mehrheitlich als ausreichend, ist also gar nicht ohne Weiteres

38 Vgl. Nick, R.: Schwesterparteien. CDU, CSU und Österreichische Volkspartei - Ein Vergleich, Innsbruck, 1984, 41.

39 Vgl. Butzlaff, F./Micus, M./Walter, F.: Im Spätsommer der Sozialdemokratie?, in: Dies. (Hg.):Genossen in der Krise?, a.a.O., 271-300.

40 Vgl. Honnigfort, B.: Kiel oben, in: Frankfurter Rundschau, 27.02.2011. 
stärker zu aktivieren. Für die SPD gilt insofern dasselbe wie für ihre österreichische Schwesterpartei, für die im Jahr 1992 Mitarbeiter der oberösterreichischen Landesgeschäftsstelle diagnostizierten, dass die Möglichkeiten der Basismitbestimmung deshalb nicht ausreichen würden, ,weil sie von der ,Basis“ viel zu wenig in Anspruch genommen werden“. Tatsächlich wäre viel erreicht, ,wenn die bestehenden Mitbestimmungsmöglichkeiten von den Mitgliedern und Funktionären ausgeschöpft würden.“41

Dennoch hält das „Organisationspolitische Grundsatzprogramm der SPD“, das auf dem Bundesparteitag im Dezember 2011 verabschiedet werden soll, an der Ausweitung der Partizipationsmöglichkeiten für die Mitglieder fest. Interessant ist dagegen, dass die Parteiführung von ihrem ursprünglichen Vorhaben, auch Nicht-Mitglieder in Form von „offenen Vorwahlen“ an der Personalauswahl zu beteiligen, mittlerweile Abstand genommen hat und dieses Beteiligungselement in der Beschlussvorlage nicht mehr enthalten ist. ${ }^{42}$ Von Anfang an standen die vor allem vom SPD-Vorsitzenden Sigmar Gabriel propagierten offenen Vorwahlen in einem schroffen Widerspruch zu den als Ergänzung der bereits bestehenden Quotierungen vorgesehenen „Migrantenquote“, die ebenfalls von Gabriel gefordert wurde und künftig 15 Prozent der Ämter in den Führungsgremien der Bundespartei zugunsten von Migranten dem freien Besetzungswettbewerb entzieht.

Dagegen beinhaltet die beabsichtigte Stärkung der Ortsvereinsebene beträchtliche Chancen, wie der europäische Vergleich zeigt. Kümmererparteien befinden sich international im Aufwind. Über die lokale Ebene kann Vertrauen hergestellt werden, hier begegnen die Parteien den Bürgern konkret, durch die Ortsvereinsarbeit können nicht zuletzt verbreitete Ressentiments und der Eindruck der Abgehobenheit von Parteien aufgelöst werden. Dazu müssen die Ortsvereine den Bürgern bei konkreten Problemen als Ansprechpartner zur Verfügung stehen und im Alltagsleben wahrnehmbar, präsent, sichtbar sein. Das kann mittelbar geschehen, über zunächst unpolitisch erscheinende Aktivitäten und Veranstaltungen wie in Schweden mit den Volkshäusern, die vielerorts als Kulturklubs, Jugendhäuser und Freizeitstätten fungieren -, oder direkt durch Hausbesuche, wie sie modellhaft in einigen norwegischen Kommunen durchgeführt wurden. Auch die im letzten Wiener Landtagswahlkampf von der SPÖ praktizierte intensive Prä-

41 Buchinger, R./ Stadler, F.: Thesenpapier zur Parteireform. Innerparteiliche Organisation - Innerparteiliche Demokratie, Linz, 1992, 5.

42 Vgl. Carstens, P.: Näher ran an die Realität, in: Frankfurter Allgemeine Zeitung, 21.09.2011, 4. 
senz in den Wohnungen des Gemeindebaus durch Mediatoren, Ordnungshüter und Ansprechpartner war erfolgreich. Auch dies dürfte einen beträchtlichen Anteil daran gehabt haben, dass die Wiener Sozialdemokraten im Jahr 2010 vor dramatischen Einbrüchen in den Gemeindebauten der Hauptstadt - wie anderthalb Jahrzehnte zuvor geschehen - bewahrt wurden, obwohl die Rechtspopulisten ein ähnlich hohes Gesamtergebnis wie 1996 erzielten. ${ }^{43}$

Der Landtagswahlkampf 2010 der SPÖ zeigt in jedem Fall das elementare Erfordernis von Balance. Politik ist - zumal in föderalen Bundesstaaten und pluralistischen Volksparteien - die Orchestrierung von Gegensätzlichkeiten, das Management von Heterogenitäten. Auch Parteireformer müssen immer das Gleichgewicht halten: zwischen Tradition und Moderne, zwischen der Berufspolitikerpartei in den Führungsgremien und der Freiwilligenorganisation an der Basis, zwischen einer Strategie der professionellen Stimmenmaximierung und der Pflege der Geselligkeits- und Identifikationsbedürfnisse bei den Stammanhängern. Insofern darf auch nicht vollständig auf mobilisierende inhaltliche Perspektiven, politische Visionen oder - das Balance-Erfordernis begrifflich fassend - auf „konkrete Utopien“ verzichtet werden. Wie gesagt, die Parteiorganisation darf nicht Selbstzweck sein, sie muss Instrument sein, mit und für Menschen diese Gesellschaft zu verändern. Die Sozialdemokraten müssen sich also zunächst einmal klar darüber werden, was sie auf welchem Wege und mit welchen Mitteln für welche Zielgruppe zu erreichen wünschen, wohin sie wollen, wie und mit wem sie dorthin gelangen möchten.

\section{Volksparteilicher Sinnbedarf}

Das ist durchaus nicht so selbstverständlich, wie es sich womöglich anhört. Stets hat man die Volksparteien als einen postideologischen Parteientypus betrachtet. Doch mit dieser Sichtweise lag man falsch. Denn die Volksparteien lebten gerade von den Residuen der alten Weltanschauungsmilieus, die den Integrationsstoff für die nachtraditionalen Bündnisse lieferten und die Mobilisierungsenergien bereitstellten, die eine Zeit lang noch farbiges und wurzelträchtiges Personal für die politische Repräsentation zulieferten. Lange wurden die Volksparteien dafür gerühmt, dass sie unterschiedliche Gruppen und Einstellungen bündeln konnten und politisch elastisch zu operieren verstanden. Das aber gelingt ihnen, auch den Sozialdemokraten, zunehmend weniger. Denn die Fähigkeit zur Bindung und

43 Vgl. Apfl, S./Toth, B.: Blues statt Blut. Heinz-Christian Straches FPÖ schwächelt. Haben die Großparteien im Umgang mit dem Rechtspopulisten dazugelernt?, in: Falter, 22.09.2010. 
Beweglichkeit hängt stets daran, dass Parteien über einen inneren Kern von Normen und Überzeugungen verfügen, über verlässliche Loyalitäten und konstante Anhänglichkeiten durch weltanschauliche Überlieferung. In dem Moment, als die Ströme der Tradition versiegten, ging die Zeit der modernen Volksparteien, auch ihrer sozialdemokratischen Variante, zu Ende. ${ }^{44}$ Mit der Erschlaffung der Weltanschauungen und der Auflösung der sie tragenden Subkulturen setzte ebenfalls der Verschleiß der Volksparteien ein.

So fällt es schwer, die SPD noch großzügig mit dem Zertifikat „Volkspartei“ zu evaluieren. Als intakte Volkspartei, die sie erst in den 1960er Jahren wurde, verknüpften die Sozialdemokraten in ihrer Führung Menschen grundverschiedener Generationen und Lebenserfahrungen: den skandinavischen Emigranten, den Leutnant der Wehrmacht, den Moskauer Kommunisten, den Widerständler der bekennenden Kirche. In Wahlkämpfe und Kabinette zogen sie mit seinerzeit modernen Gewerkschaftern wie Georg Leber, zugleich Ende der 1960er Jahre Mitglied im Zentralkomitee der deutschen Katholiken, oder Walter Arendt; mit Vertretern der evangelischen Kirche wie Jürgen Schmude, Gustav Heinemann, Erhard Eppler und Johannes Rau sowie mit redegewandten und ideenreichen Groß- und Bildungsbürgern wie Carlo Schmid, Horst Ehmke und Karl Schiller; mit strategiebegabten Konzeptionalisten der Außenpolitik wie Egon Bahr und experimentell couragierten Unternehmern wie Philip Rosenthal. Verglichen mit diesem Erfolgsjahrzehnt der volksparteilichen Wandlung klafft in der SPD heute ein riesiges Repräsentationsloch. Auffallend ist überdies, dass in der SPD seit einigen Jahren schon kein herausragender Sozialpolitiker, kein hochkarätiger Wissenschafts- und Kulturpolitiker, vor allem kein konzeptionell denkender Außenpolitiker und natürlich auch kein berufserfahrener Wirtschaftspolitiker für die mittlere Zukunft mehr sichtbar wird.

Doch kann man auch einen anderen Blickwinkel einnehmen, bei dem sich die Lage der Sozialdemokratie weniger traurig ausmacht. So hat die Ende 2006 viel beachtetet Studie von TNS Infratest Sozialforschung zur „Gesellschaft im Reformprozess“ ergeben, dass die SPD - nahm man nur den Querschnitt der Repräsentanz - die ausgewogene Volkspartei auf mittlerem Niveau schlechthin war. Zumindest war sie in allen neun von Infratest identifizierten Milieus der deutschen Gesellschaft mit über 25 Prozent, bei einer Ausnahme gar mit über 30

44 Vgl. auch Walter, F.: "Renovation totale”?, in: Rüttgers, J. (Hg.): Berlin ist nicht Weimar. Zur Zukunft der Volksparteien, Essen, 2009, 139-145. 
Prozent der Wähler vertreten. ${ }^{45}$ Der Union gelang (und gelingt) das nicht mehr gleichermaßen flächendeckend, den übrigen Parteien erst recht nicht. Die SPD umwölbt mehr Spektren - von oben bis unten, von jung bis alt, von Gebildeten bis Ungebildeten, von Etatisten bis Marktfans - als der christdemokratische Rivale. Nur ist der Bogen, den sie dabei zu schlagen hat, denkbar weit dimensioniert; und darin wurzeln, wie aufgezeigt, etliche der Probleme der Partei. Soziale und kulturelle Dehnung bedeutet eben Vorzug wie Belastung zugleich.

Will eine Partei mit einem derart vielschichtigen Wählerreservoir wie die SPD sich auf lediglich eine zentrale Figur an der Spitze beschränken, dann müsste diese Person eine immens facettenreiche Gestalt sein, müsste als Projektionsfläche für verschiedene Bedürfnisse, Einstellungen und Kulturen taugen, müsste rochieren, sich neuen Verhältnissen blitzschnell anpassen. Schröder war die Verkörperung eines solchen Versuchs; den Sozialdemokraten ist das bekanntermaßen nicht nur gut bekommen. Es war kein Zufall, dass die SPD gerade auch in ihren besten Zeiten ein Triumvirat bzw. eine Troika an ihrer Spitze hatte, bestehend aus Herbert Wehner, Willy Brandt, anfangs Fritz Erler, später dann Helmut Schmidt. Jeder einzelne war ein gewichtiges Kaliber. ${ }^{46}$ Dennoch gelang auch ihnen die volksparteiliche Integration nur durch kooperative Führungsvielfalt. Allem Anschein nach orientiert sich die gegenwärtige SPD wieder an diesem Modell, nun in Gestalt des Trios Sigmar Gabriel, Frank-Walter Steinmeier und Peer Steinbrück, die von unterschiedlichem Temperament sind und differente Wählermilieus ansprechen sollen, wobei sich der Parteivorsitzende in erster Linie nach innen wenden, die eigenen Truppen beieinander halten, bei Bedarf aktivieren, auch auffrischen und orientieren soll.

\section{Wiedererstarkte Sozialdemokraten?}

Zwei Jahre nach der bitteren Bundestagswahlniederlage von 2009 wirkt die SPD wieder deutlich erholt. Bis zur Mitte der gegenwärtigen Legislaturperiode fanden in der Republik insgesamt acht Regionalwahlen statt. ${ }^{47}$ In fünf Bundesländern

45 Vgl. Neugebauer, G.: Politische Milieus in Deutschland. Die Studie der Friedrich-Ebert-Stiftung, Bonn, 2007.

46 Vgl. Rupps, M.: Troika wider Willen. Wie Brandt, Wehner und Schmidt die Republik regierten, Berlin, 2004.

47 Hierzu und im folgenden die Angaben der von der Konrad-Adenauer-Stiftung herausgegebenen Wahlanalysen: Neu, V.: Landtagswahlen in Nordrhein-Westfalen, Berlin, 2010; ders.: Bürgerschaftswahl in Hamburg am 20. Februar 2011, Berlin, 2011; ders.: Landtagswahl in Sachsen-Anhalt am 20. März 2011, Berlin, 2011; ders.: Landtagswahl in Baden-Württemberg am 27. März 2011, Berlin, 2011; ders.: Landtagswahl in Rheinland-Pfalz am 27. März 2011, Berlin, 2011; ders.: Bürgerschaftswahl in Bremen am 
(Sachsen-Anhalt, Rheinland-Pfalz, Bremen, Mecklenburg-Vorpommern und Berlin) konnte die SPD ihre Regierungsposition behaupten, in drei Bundesländern (Nordrhein-Westfalen, Hamburg und Baden-Württemberg) gelangte sie aus der Opposition heraus in das Kabinett. Aus sozialdemokratischer Sicht stellt sich diese Bilanz höchst erfreulich dar. Doch schaut man näher hin, dann lässt sich rasch erkennen, dass Stabilisierung und Ausbau sozialdemokratischer Regierungsmacht in den Bundesländern vor allem eine Folge koalitionspolitischer Vorzüge sind, da die SPD mit den Grünen einen primären Allianzpartner hat, der zuletzt kräftige Zuwächse aus dem Lager von CDU und FDP verzeichnete, während die Christdemokraten aufgrund der enormen Schwäche der FDP seit Ende 2009 immer mehr an der Aufgabe einer Mehrheitsbildung des altbürgerlichen Lagers gescheitert sind.

Mit einer üppigen Ausdehnung der eigenen Wählerbasis konnten die Sozialdemokraten, sieht man von den Wahlgängen in Hamburg und MecklenburgVorpommern ab, dagegen nicht brillieren. Die SPD hielt in Sachsen-Anhalt und Bremen in etwa ihr vorangegangenes Ergebnis. Dagegen hatte sie einen weiteren Rückgang in Nordrhein-Westfalen, Rheinland-Pfalz (-9,9 Prozentpunkte!), Baden-Württemberg und Berlin zu verkraften. Doch im Vielparteiensystem können elektorale Verlierer, wenn sie im Spiel der Mehrheitsbildung einen günstigen Ort einnehmen, gleichwohl machtpolitisch hinzugewinnen. Das ist in den beiden fraglos bedeutsamen Bundesländern Baden-Württemberg und NordrheinWestfalen geschehen. Nimmt man diese beiden Länder wahlanalytisch schärfer ins Visier, dann kommen einige Charakteristika des gegenwärtigen sozialdemokratischen Zustands trefflich zum Vorschein.

Die Sozialdemokraten an Rhein, Ruhr und Lippe verschlechterten sich 2010 gegenüber 2005 - als die Partei des Ministerpräsidenten Peer Steinbrück gegen die CDU Jürgen Rüttgers ' mit Aplomb verlor - um weitere 2,6 Prozentpunkte auf 34,5 Prozent. Die Anteile der baden-württembergischen Sozialdemokraten sind 2011 um zwei Prozentpunkte auf 23,1 Prozent zurückgegangen. Die SPD in NRW ist damit auf den Stand von 1954 zurückgefallen. Die Sozialdemokraten im Südwesten fuhren 2011 gar das schlechteste Ergebnis in der Geschichte des Bundeslandes ein. Zuwächse verbuchte die SPD in diesen beiden Ländern in alterstruktureller Perspektive allein bei den über 60-Jährigen, erwerbsanalytisch betrachtet lediglich bei den Rentnern. Bei den Wählern, die im Beruf standen,

22. Mai 2011, Berlin, 2011; ders.: Landtagswahl in Mecklenburg-Vorpommern am 4. September 2011, Berlin, 2011; ders.: Abgeordnetenhauswahl in Berlin am 18. September 2011, Berlin, 2011. 
konstatierten die Forschungsinstitute unisono ein Minus von 3,9 Prozent. In der Altersgruppe der 18-44-Jährigen fiel der Abfluss von Wählern mit über sechs Prozent am deutlichsten aus. Bemerkenswert ist die Differenz, die sich zwischen den Sozialdemokraten in Baden-Württemberg und denen in Nordrhein-Westfalen im Resonanzbereich der manuellen Arbeiter auftat. Die Partei von Hannelore Kraft hatte bei den Arbeitern mit einem Plus von ein Prozent zumindest etwas Boden gut gemacht. Die seit Jahrzehnten besonders mittelschichtaffinen Sozialdemokraten von Nils Schmidt stießen hingegen im Unterschichtsektor auf zusätzliche Ablehnung (mit einem Minus von sechs Prozentpunkten bei den Arbeitern und neun Prozentpunkten bei Arbeitslosen). Schließlich: Der SPD kommen insgesamt, nicht zuletzt aufgrund der Hochs der Grünen, die formal Hochgebildeten sukzessive abhanden. Die Partei wird folglich auch in ihrer Bildungsstruktur „mittiger“, da sich der Überschuss an Geringgebildeten bereits zwischen 2003 und 2009 markant abgetragen hatte.

Die Trends, die hier erhoben und referiert worden sind, gelten zumindest für die alten Bundesländer allgemein. Die Gruppe, welche die SPD am stärksten trägt, bilden die Rentner (vgl. Abb. 2), in einem früher nicht gekannten Ausmaß gerade die weiblichen. Gravierende Einbrüche sind hingegen bei den Wahlbürgern, die sich in der Ausbildung befinden, zu entdecken. Im Westen profitieren davon die Grünen, im Osten stärker noch die NPD. In Sachsen-Anhalt rekrutierte die NPD bei den jüngsten Landtagswahlen mit 14,6 Prozent mehr 18-29-jährige Männer als die SPD, die nur auf 14,3 Prozent kam. Einen Hinweis auf die mögliche $\mathrm{Zu}-$ kunft der Sozialdemokratie und der Parteien allgemein mag auch das Ergebnis der Berliner Wahlen zum Abgeordnetenhaus Mitte September 2011 geben. Auch

Abbildung 2: SPD-Wähler nach Alter

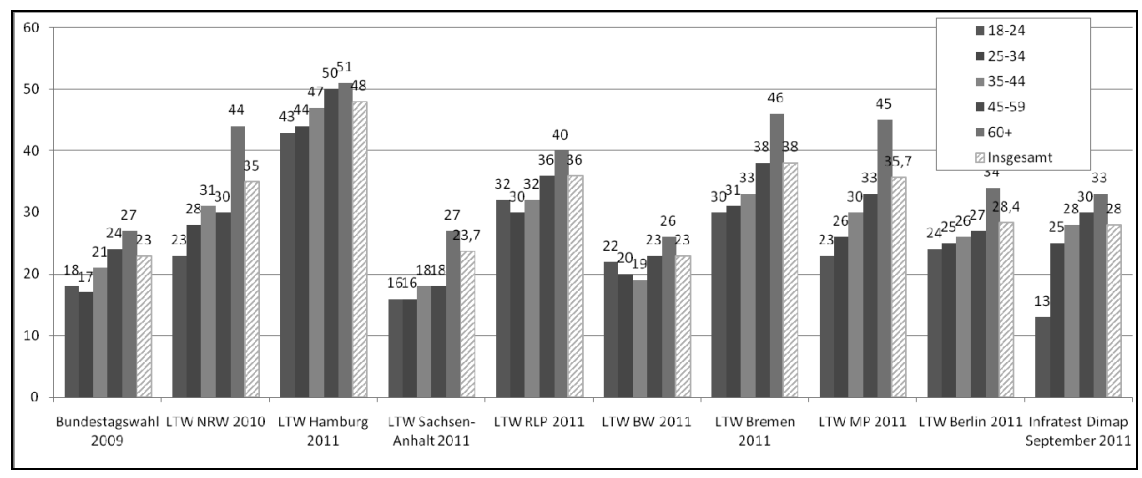

Quelle: Eigene Darstellung auf Basis der Zahlen von Infratest Dimap. 
hier stoßen wir bei den 18-29-Jährigen auf die größten Verluste für die SPD (mit einem Minus von 7,3 Prozent). Die Grünen konnten das sozialdemokratische Minus in dieser Gruppe kaum kompensieren. Dafür holten die Piraten bei den 18-29-jährigen Männern 19,4 Prozent der Voten und kamen aus dem Stand nah an die 24,8 Prozent sozialdemokratischer Anteile in dieser Kohorte heran. Eine Über-30-Prozent-Partei ist die SPD in der deutschen Hauptstadt lediglich bei der Kohorte 60plus, auch hier: besonders stark im weibliche Segment.

Insgesamt haben also die Grünen am stärksten aus dem Reservoir vormaliger sozialdemokratischer Wähler geschöpft. Bei der Bundestagswahl 2009 zog noch DIE LINKE (mit 1.100.000 Stimmen) mit Abstand den größten Nutzen aus dem abtrünnigen Teil des sozialdemokratischen Potentials. Noch kräftiger fiel der sozialdemokratische Aderlass (mit einem Minus von 2.040.000 Stimmen) in Richtung Nichtwähler aus. Der Exodus zu den Nichtwählern ist seit 2009 gestoppt; die Konversion zu den Linken zumindest gedrosselt. Dafür ist der Übergang zu den Grünen erheblich. Allerdings haben die Sozialdemokraten im Gegenzug umfangreiche Zugewinne aus der Anhängerschaft der CDU erreichen können. Und was an DIE LINKE abging, ließ sich durch freidemokratische Zuzügler wettmachen (vgl. Abb. 3). Kurzum: Die Wählerschaft der SPD im Zeitraum vom September 2009 bis September 2011 scheint deutlich weniger links und grün orientiert, dürfte dafür eine stärker liberal-konservative Erwartungshaltung an Politik erfahren haben.

Abbildung 3: SPD-Wählerwanderungen nach der Bundestagswahl 2009

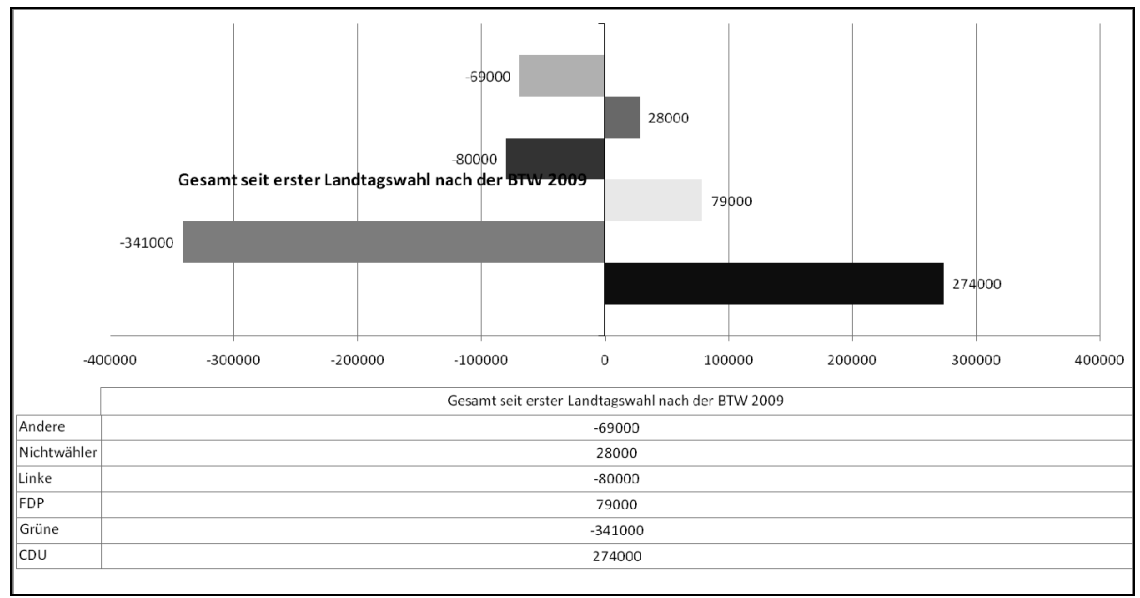

Quelle: Eigene Berechnung aus den Zahlen von Infratest Dimap. 


\section{Neue Diskurse}

Es ist dieser Wandel der Anhängerschaft, der in Zukunft in der deutschen Sozialdemokratie Debatten unterfüttern wird, wie sie seit einigen Jahren vorzugsweise in Großbritannien und - über internationale Diskussionszirkel vermittelt, wenngleich mit geringerer Intensität - in letzter Zeit auch in Deutschland geführt werden. Die Vorreiterrolle der britischen Labour-Partei ergibt sich nicht zuletzt daraus, dass hier auch die Konservativen, ihrerseits angetrieben von den Erfolgen von New Labour unter Tony Blair, intensiv an Neudefinitionen ihres gesellschaftlichen Ortes, ihrer politischen Rolle und perspektivischen Ziele feilen. Zumal die Konservativen mit ihrer progressiven Agenda der rechten Mitte, zu deren Kernelementen die Verabschiedung eines dogmatischen Wirtschaftsliberalismus, das Bekenntnis zum regulativen Wohlfahrtsstaat sowie die Hinwendung zu grünen Umweltthemen gehörten, jüngst den Regierungswechsel geschafft haben. ${ }^{48}$ In Reaktion auf diesen modernisierten und sozialdemokratisierten Konservatismus - treffend als „Red Toryism“ bezeichnet - entwickelten mit der Labour Party verbundene Sozialwissenschaftler das Gegenmodell eines durch konservative Werte angereicherten Sozialdemokratismus, den sie konsequenterweise „Blue Labour" titulierten.

In engem Zusammenhang mit „Blue Labour“ steht der Diskurs über die „Gute Gesellschaft" (good society), der sich vor rund sechs Jahren auf einer Tagung britischer Sozialdemokraten entspann. Durch ein gemeinsames Papier des Labour-Abgeordneten Jon Cruddas und der SPD-Generalsekretärin Andrea Nahles erhielt er zusätzlichen Schwung und wird seither unter Beteiligung der FriedrichEbert-Stiftung und dem Think Tank Compass insbesondere auf der Internetseite des Social Europe Journal geführt. Obwohl beide Debatten nicht identisch sind, weisen sie doch zahlreiche Überschneidungen bei den beteiligten Personen und propagierten Werten auf, bei Problemdiagnosen wie auch skizzierten Lösungsvorschlägen der sozialdemokratischen Krise. ${ }^{49}$

Vor dem Hintergrund der Wirtschafts- und Finanzkrise hat sich - wenig verwunderlich - das Bild, das sich die Sozialdemokraten von den freien Kräften des Marktes machen, verglichen mit dem strahlenden Optimismus der Vorjahre wieder getrübt. Offen werden vor allem der Finanzkapitalismus kritisiert und die

48 Vgl. Blond, P.: Red Tory. How Left and Right have broken Britain and how we can fix it, London, 2010.

49 Vgl. Meyer, H./Spiegel, K.-H.: Die Gute-Gesellschaft-Debatte. Wie weiter mit der Sozialdemokratie?, Berlin, 2010. 
Einhegung und Regulation der Marktkräfte diskutiert; auch Gedanken über alternative Wirtschaftsordnungen sind nicht mehr tabu. Erstaunlicher mutet schon die Staatsskepsis der Beteiligten an, auch weil ausgewiesene Parteilinke führend an den Debatten beteiligt sind. Die angestrebte „Gute Gesellschaft“ ist durchaus kein Staatssozialismus, auch der zentralistische Wohlfahrtsstaat wird in seiner Gestalt als bürokratischer Apparat kritisiert, da er auf die Vielfalt konkreter Einzelschicksale nicht angemessen reagieren könne, Eigeninitiative blockiere und die zivilgesellschaftliche Selbstorganisation hemme.

Darum aber geht es den sozialdemokratischen Konzeptdenkern vor allem: um die Förderung von Selbstorganisation. Im Zentrum stehen lokale Zusammenschlüsse und Gemeinschaften, die Menschen miteinander verbinden, sie aktivieren und ihre Gemeinsamkeiten erkennen lassen - und die grundlegenden Werte Reziprozität, Solidarität und Gegenseitigkeit mit Leben und Sinn füllen. Aus der Gemeinschaftsprärogative ergeben sich die kennzeichnenden Merkmale der „Guten Gesellschaft“. In dem Assoziationsimperativ wurzelt der Antikapitalismus der „Post-Dritte-Weg“-Sozialdemokraten, der sich aus der Sorge speist, dass die Grundlagen gemeinschaftlichen Handelns durch die ,Vermarktlichung“ sämtlicher Lebensbereiche unterminiert würden. Er begründet die Ablehnung zentralistischer Vereinheitlichung und abstrahierender Universalismen zugunsten lokaler Variationen, praktischer Gemeinschaftserlebnisse und konkreter Organisationserfahrungen; und er fundiert auch die Pluralismuskritik in den programmatischen Entwürfen, da gegenseitiges Vertrauen und Solidarität ein Übermaß an Fremdheit nicht ertragen würden.

In engem Zusammenhang mit dem Gemeinschaftsdenken steht insofern auch der Konservatismus der Ansätze sozialdemokratischer Neubegründung, die Ablehnung der technokratischen Progressivität des Dritten Weges, die Betonung von Nation und Familie sowie die Absicht, das Gute und Bewährte zu konservieren. Eben dieser Traditionalismus, bei Teilen von Labour gewiss deutlicher präferiert als derzeit in der SPD, soll wieder stärker an die deaktivierten klassischen Kerne heranführen, zumal die Bezüge auf einen ursprünglichen, frühindustriellen und von den Mitte-Rechts-Parteien vergessenen Konservatismus ein Bekenntnis zum Arbeitsethos des Facharbeitertums und zu einem breitgestreuten Volkseigentum einschließen - inklusive nostalgischer Erinnerungen an die Arbeiterklassensoli- 
darität in der Frühzeit der Bewegung, als sich die sozialdemokratische Bewegung auf ein dichtes Organisationsnetz stützen konnte. ${ }^{50}$

Die Visionen von der „Guten Gesellschaft“ sind aber auch für die Mittelschichten und Bessergebildeten attraktiv, aus deren Reihen Forderungen nach stärkerer Basisbeteiligung und Bürgerpartizipation am lautesten erhoben werden. Die „Gute Gesellschaft“ soll sich nicht zuletzt durch mehr Mitbestimmung auszeichnen, die assoziierten Bürger sind als effektive Gegenmacht gegen übermächtig scheinende Marktkräfte und Staatsbürokratien gedacht. ${ }^{51}$ Daraus ergibt sich ein ganz modernes Verständnis von Gerechtigkeit, die sich letztlich nicht in der Chancengleichheit oder einer gerechten Verteilung des Wohlstandes erschöpft. Vielmehr will sie die Bürger von ungewollter Fremdbestimmung befreien, ihnen die Kontrolle über das eigene Leben zurückgeben und Staatseingriffe auf solche Bereiche und Anlässe beschränken, für die die Regierenden von aktiv sich einmischenden Bürgern ermächtigt worden sind. Zudem: Das Parteienbild der „Guten Gesellschaft" ist in etwa das der in jüngster Vergangenheit europaweit vielversprechend erprobten Kümmererparteien.

Jedenfalls zeigt die Debatte über die „Gute Gesellschaft“ (und „Blue Labour"), dass die Sozialdemokraten zuletzt wieder ein größeres Verständnis für die Notwendigkeit von Balancen entwickelt haben: zwischen Pragmatismus und Visionen, Traditionalität und Moderne, Sicherheit und Öffnung. Die Betonung selbstorganisierten Handelns, die Besetzung von Werten wie Familie und Heimat sowie das Bewusstsein von den Gefahren zu weitgehender kultureller Heterogenität lässt Möglichkeiten erahnen, wie den Sozialdemokraten das Management des Widersprüchlichen und der erfolgsnotwendige Spagat zwischen unterschiedlichen Wählermilieus in der Zukunft wieder gelingen könnte.

50 Vgl. Glasman, M.: Labour as a radical tradition, in: Glasman, M. u.a. (Hg.): The Labour Tradition and the Politics of the Paradox, Oxford, 2011, 14-34.

51 Vgl. Cruddas, J./Nahles, A.: Die gute Gesellschaft. Das Projekt der demokratischen Linken, in: Der Freitag, 08.04.2009; Rutherford, J.: The Future is Conservative, in: Glasman, M. u.a. (Hg.): The Labour Tradition, a.a.O., 88-105. 